\title{
Triagem fitoquímica e avaliação das atividades trombolítica e citotóxica de Cecropia hololeuca Miq. (Urticaceae), Lippia alba (Mill.) N.E.Br. ex P. Wilson (Verbenaceae) e Zanthoxylum rhoifolium Lam (Rutaceae)
}

Phytochemical screening, thrombolytic and citotoxic activity evaluation of Cecropia hololeuca Miq. (Urticaceae), Lippia alba (Mill.) N.E.Br. ex P. Wilson (Verbenaceae) and Zanthoxylum rhoifolium Lam (Rutaceae)

Recebido em: 06/11/2015

Aceito em: 24/01/2016
João Victor Dutra GOMES'; Rafael Destefani FAITANIN'; Beatriz Gonçalves BRASILEIRO²; Dâmaris SILVEIRA ${ }^{3}$; Claudia Masrouah

JAMAL ${ }^{1}$

${ }^{1}$ Departamento de Ciências Farmacêticas, Centro de Ciências da Saúde, Universidade Federal do Espírito Santo. Avenida Marechal Campos, 1468, Maruípe, Vitória, ES. CEP 29043-900. Brasil. ${ }^{2}$ Instituto Federal de Ciência e Tecnologia do Sudeste de Minas Gerais, Avenida Monteiro de Castro, 550. Barra, Muriaé, MG. CEP 36880-000. Brasil. ${ }^{3}$ Faculdade de Ciências da Saúde. Universidade de Brasília. Campus Universitário Darcy Ribeiro, Asa Norte, Brasilia, DF. CEP 70910-900.Brasil.E-mail: cmjamal@gmail.com

Cecropia hololeuca, Lippia alba, and Zanthoxylum rhoifolium are plant species naturally occurring in Brazil, from Atlantic Forest, with medicinal purposes, like antihypertensive action, digestive, and treatment of malaria, respectively. In this study, we evaluated the chemical profile, cytotoxicity, and thrombolytic potential of the ethanol extract of the leaves of these plant species. The phytochemical profile was characterized by phytochemical tests of metabolites classes identification. The preliminary toxicity of the extracts was determined by brine shrimp lethality bioassay and the in vitro thrombolytic activity was evaluated through the extracts capacity to cause lysis in human blood clot. Phytochemicals tests indicated the presence of flavonoids and alkaloids in the three extracts. Saponins, triterpenes, and naphthoquinones were only positive for C. hololeuca. Cecropia hololeuca did not show cytotoxicity to Artemia salina $\left(\mathrm{LD}_{50}>1000 \mathrm{ppm}\right)$ while Z. rhoifolium presented $\mathrm{LD}_{50}$ equal to $719.44 \mathrm{ppm}$ and $L$. alba $<250 \mathrm{ppm}$, which indicates attention to the safety of this medicinal plant use. The thrombolytic activity of $L$. alba and C. hololeuca was $6.43 \pm 2.08$ and $9.64 \pm 1.83 \%$, respectively, showing a small potential. Z. rhoifolium lysis achieved $24.71 \pm 10.52 \%$, indicating promising activity $(\mathrm{p}<0.001)$. The thrombolytic activity of $Z$. rhoifolium justifies further studies to investigate the components responsible for this activity. This study, faced with the literature, is the first report assessing the thrombolytic activity of these plant species. The findings of this study contribute to the chemical-biological knowledge of these species.

Keywords: thrombolytic activity; medicinal plants; phytochemistry

\section{RESUMO}

Cecropia hololeuca, Lippia alba, e Zanthoxylum rhoifolium são espécies vegetais de ocorrência natural no Brasil, características de regiões de Mata Atlântica e, dentro de suas finalidades medicinais, estão incluídas ação anti-hipertensiva, digestiva e no tratamento de malária. Neste estudo, foram avaliados o perfil fitoquímico e as atividades citotóxica e trombolítica do extrato etanólico das folhas destas espécies vegetais. O perfil químico dos extratos foi obtido por meio de ensaios fitoquímicos clássicos, a fim de identificar as classes químicas presentes. A toxicidade preliminar foi avaliada frente Artemia salina e a atividade trombolítica foi determinada in vitro a partir da lise de coágulo de sangue humano. Os testes fitoquímicos indicaram a presença de alcaloides e flavonoides nos três extratos investigados. Saponinas, triterpenos e naftoquinonas foram detectados apenas em C. hololeuca. Essa não demonstrou toxicidade frente Artemia salina $\left(\mathrm{DL}_{50}\right.$ $>1000 \mathrm{ppm}$ ), enquanto Z. rhoifolium apresentou $\mathrm{DL}_{50}$ igual a $719,44 \mathrm{ppm}$ e $L$. alba $<250 \mathrm{ppm}$, o que indica atenção quanto à segurança no uso desta planta medicinal. A atividade trombolítica de $L$. alba e C. hololeuca foi de 6,43 $\pm 2,08$ e 9,64 $\pm 1,83 \%$, respectivamente, mostrando baixa atividade. Por sua vez, Z. rhoifolium alcançou lise de 24,71 $\pm 10,52$ $\%$, indicando promissora atividade $(\mathrm{p}<0,001)$. A atividade trombolítica de $Z$. rhoifolium justifica novos estudos, a fim de investigar os componentes responsáveis pela atividade. Perante a literatura, este é o primeiro relato da avaliação da atividade trombolítica destas espécies vegetais. Os resultados encontrados neste trabalho contribuem para o conhecimento químico-biológico das respectivas espécies.

Palavras-chave: atividade trombolítica; plantas medicinais; fitoquímica 
INTRODUÇÃO

As plantas medicinais foram as primeiras alternativas de tratamento de diversas enfermidades $(1,2)$. A potencialidade das espécies vegetais como fonte de substâncias úteis no desenvolvimento de medicamentos faz com que o interesse em produtos naturais seja cada vez maior, tornando as pesquisas com plantas medicinais ainda mais bem vistas $(3,4)$. A Organização Mundial da Saúde estimou que mais de 60 bilhões de dólares sejam investidos em produtos naturais anualmente (5) e, aproximadamente, $80 \%$ da população mundial confia na medicina tradicional para cuidados primários a saúde (3). Neste sentido, faz-se necessário conhecer a potencialidade químico-biológica de plantas medicinais, com o objetivo de racionalizar o seu uso.

Uma das estratégias empregadas para a realização de pesquisas em plantas medicinais é a utilização de espécies com uso terapêutico já atribuído por uma determinada população - conhecimento etnofarmacológico (6). Estudos com extratos de espécies vegetais repercutem diretamente em pontos básicos, como: desenvolvimento de medicamentos; asseguração do uso de plantas medicinais pela população, com a confirmação de eficácia e segurança, bem como atribuição de possíveis novos usos terapêuticos; contribuição ao conhecimento da memória químico-biológica de espécies vegetais (6).

A atividade trombolítica de produtos naturais tem sido avaliada por pesquisadores, considerando o método proposto por Prasad e cols (2006). Fármacos trombolíticos são úteis no tratamento de patologias, como trombose venosa cerebral (7). Algumas espécies vegetais já foram investigadas sob este ponto de vista, tais como Bacopa monnieri, Fagonia arabica e Cestrum diurnum, mas ainda não está estabelecido se há correlação de algum grupo químico que desempenhe tal atividade (8-9).

Eventos observados após o uso clínico de medicamentos trombolíticos usuais, como hipotensão e acidente vascular hemorrágico cerebral, evocam a necessidade de buscar novos protótipos para esta classe. Além disso, pacientes com idade superior a 65 anos, com prévia ressuscitação cardiorrespiratória, e indivíduos diabéticos, por exemplo, são consideradas restrições clínicas para a utilização destes medicamentos (10-12). A investigação de plantas medicinais sob esta óptica pode levar ao descobrimento de potenciais moléculas de interesse biológico.

A Mata Atlântica é considerada um hotspot de biodiversidade. Neste bioma, muitas espécies já se encontram ameaçadas de extinção e as mudanças climáticas tendem a comprometer o acervo biológico de fauna e flora, fazendo com que os estudos neste bioma sejam ainda mais urgentes (13). Muitas vezes, espécies reconhecidas pelo caráter medicinal são retiradas do seu ambiente natural, sem qualquer garantia de sustentabilidade. Eugenia multicostata (Myrtaceae), por exemplo, conhecida popularmente como araçá-piranga foi incluída na lista de plantas ameaçadas de extinção da FAO (Organização das Nações Unidas para a Agricultura e a Alimentação) em 2001 (14).

Cecropia hololeuca Miq. (Urticaceae), Lippia alba (Mill.) N.E.Br. ex P. Wilson (Verbenaceae) e Zanthoxylum rhoifolium Lam (Rutaceae) são três representantes vegetais da Mata Atlântica, com uso popular medicinal conhecidos (15-20). A espécie $C$. hololeuca, conhecida como "embaúba", é encontrada nos Estados da região Sudeste e no Estado da Bahia (15); e o uso das folhas é descrito em casos de hipertensão e bronquite. O pó das folhas e dos ramos novos é utilizado em casos de hipertensão, enquanto a decocção das folhas tem uso terapêutico em pacientes com bronquite (16). Esta espécie também é encontrada em regiões de Cerrado e é endêmica no Brasil (15).

Também conhecida como "cidreira" e "erva-cidreira", L. alba é encontrada em todos os Estados do Brasil. Além da Mata Atlantica, pode ser também encontrada em regiões de Caatinga, Cerrado e Amazônia (17); e entre seus usos populares, podem ser citados ação digestiva e em casos de dores de barriga (18).

Zanthoxylum rhoifolium é conhecida popularmente como "espinho-de-vintém", "mamica-de-porca", "juva", "tinguaciba", entre outros, e é distribuída por todo Brasil (19). Jullian e cols (2006) realizaram um estudo sobre esta espécie, validando seu uso tradicional na prevenção e tratamento da malária (20). Neste estudo, foi observado que os alcaloides de Z. rhoifolium podem ser os compostos responsáveis por esta propriedade, os quais podem ser obtidos a partir da decocção da casca desta espécie.

Diante do exposto, o objetivo deste trabalho foi avaliar o perfil químico do extrato etanólico das folhas de C. hololeuca, L. alba, e Z. rhoifolium, bem como as atividades citotóxica frente a Artemia salina e trombolítica in vitro.

\section{MATERIAIS E MÉTODOS}

Coleta e identificação do material vegetal e preparação dos extratos vegetais. As folhas de Cecropia hololeuca, Lippia alba e Zanthoxylum rhoifolium foram coletadas no município de Governador Valadares, Minas Gerais, Brasil. O material vegetal foi devidamente identificado, com o depósito de exsicata no Herbário da Universidade Vale do Rio Doce (UNIVALE), sob número de identificação 802 para C. hololeuca, 621 para L. alba e 901 para Z. rhoifolium.

As folhas foram secas por aproximadamente 72 horas, em estufa a $38-40{ }^{\circ} \mathrm{C}$. O material vegetal foi pulverizado em moinho de facas e extraído, separadamente, por maceração com etanol durante 15 dias, com renovação de solvente a cada 5 dias. Em seguida, o solvente foi eliminado, com auxílio de rotaevaporador. Os três extratos obtidos foram conservados em refrigerador $\left(2\right.$ a $\left.5^{\circ} \mathrm{C}\right)$ até sua utilização nos ensaios químico e biológicos subsequentes. 
Triagem fitoquímica preliminar. Os extratos etanólicos obtidos foram submetidos à triagem fitoquímica preliminar para detecção das principais classes de metabólitos secundários. Nestes testes foi avaliada a presença de flavonoides, triterpenos, esteroides, naftoquinonas, saponinas, alcaloides, taninos, cumarinas e heterosídeos antracênicos (21-23).

Avaliação da citotoxicidade frente à Artemia salina Leach. A citotoxicidade dos extratos em estudo foi avaliada frente à Artemia salina (24), de acordo com o método de Meyer e cols (1982). Ovos encistados de A. salina foram incubados por 48 horas em solução salina ( $36 \mathrm{~g} / \mathrm{L})$ a $28{ }^{\circ} \mathrm{C}$, sob luz e aeração constantes, para sua eclosão e obtenção das larvas. As larvas foram, então, distribuídas em tubos de ensaio contendo os extratos em diferentes concentrações $(250,500$ e 1000 ppm). Como controle positivo de letalidade, foi utilizado dicromato de potássio $(25,50$ e $100 \mathrm{ppm}$ ). Como controle negativo, foi utilizado o veículo de solubilização dos extratos e do dicromato de potássio (dimetilsulfóxido, DMSO, a 1\% v/v).

Os tubos foram mantidos sob iluminação e, após 24 horas, as larvas sobreviventes foram contadas. Os resultados foram expressos em $\mathrm{DL}_{50}$ (dose necessária para obter metade das larvas mortas), obtidos a partir de regressão linear, com auxílio do Software Excel ${ }^{\circledR}$ (2007). O ensaio foi realizado em triplicata.

Avaliação da atividade trombolítica. Este ensaio foi aprovado pelo Comitê de Ética da Universidade Federal do Espírito Santo, Brasil, sob número de parecer de 148.873 .

A atividade trombolítica dos extratos em estudo foi avaliada conforme o método proposto por Prasad e cols (2006) (7). Pequenas porções de sangue venoso humano $(500,0 \mu \mathrm{L})$, contidas em tubos de microcentrífuga, foram incubadas a $37^{\circ} \mathrm{C}$, por 45 minutos. O coágulo formado em cada tubo foi pesado e o material líquido remanescente foi aspirado com auxílio de uma micropipeta, de modo a preservar o coágulo. Logo após, foi adicionado $150,0 \mu \mathrm{L}$ de agente trombolítico e os tubos foram incubados a $37{ }^{\circ} \mathrm{C}$ por 90 minutos. Após incubação, o fluido resultante da lise dos coágulos foi cuidadosamente aspirado e o peso dos coágulos foi novamente verificado, permitindo calcular o percentual de lise.

Os extratos foram avaliados na concentração de 1,0 $\mathrm{mg} / \mathrm{mL}$, solubilizados com propilenoglicol $15 \% \mathrm{v} / \mathrm{v}$. Como controle de atividade trombolítica, foi utilizado estreptoquinase a $100.000 \mathrm{UI}$, solubilizada com água destilada. O ensaio foi realizado em quadruplicata, e os veículos também foram avaliados quanto à atividade trombolítica. A atividade trombolítica dos extratos e da estreptoquinase foi comparada com os respectivos veículos, a partir do Teste $\mathrm{t}$ de Student com amostras independentes, utilizando o programa IBM SPSS Statistics 20, considerando diferença significativa quando $\mathrm{p}<0,01$.

\section{RESULTADOS E DISCUSSÃO}

Os resultados dos testes fitoquímicos estão indicados na Tabela 1.

Jullian e cols (2006) também identificaram a presença de alcaloides em e Zanthoxylum rhoifolium (20). Neste estudo foi justificado o uso popular desta espécie para o tratamento de malária, e a atividade foi atribuída aos alcaloides presentes nesta espécie.

O potencial antimalárico também é característica da espécie Cecropia hololeuca. Embora ainda não se conheça os componentes químicos desta espécie responsáveis por desempenhar tal atividade (25), a presença de alcaloides no extrato de $C$. hololeuca pode justificar esta atividade.

Aguiar e cols (2008) avaliaram a atividade antimicrobiana de diversos extratos de Lippia alba, contudo, não conduziram ensaios químicos para monitoramento dos possíveis componentes responsáveis por esta propriedade bioativa (26). Na triagem fitoquímica do extrato etanólico das folhas de $L$. alba foi possível identificar a presença de flavonoides e taninos. A presença destas duas classes químicas nesta espécie pode justificar a atividade antimicrobiana observada por outros autores, uma vez que os compostos fenólicos são conhecidos por tal propriedade (27).

Tabela 1: Triagem fitoquímica em extratos etanólicos de três espécies ocorrentes na Mata Atlântica: Cecropia hololeuca, Lippia alba e Zanthoxylum rhoifolium

\begin{tabular}{l|l|l|l} 
Classe química & C. hololeuca & L. alba & Z. rhoifolium \\
\hline
\end{tabular}

Alcaloides

Cumarinas

Esteroides

Triterpenos

Flavonoides

Naftoquinonas

Saponinas

Taninos

Heterosídeos

antracênicos

Classe química presente (+); Classe química ausente (-).

Os resultados obtidos na avaliação fitoquímica preliminar fornecem informações sobre as classes químicas que compõem os extratos obtidos e podem sugerir marcadores químicos para o controle da qualidade das respectivas drogas vegetais. Entretanto, vale ressaltar que ocorrem variações no perfil dos metabólitos secundários de espécies vegetais, as quais têm sido atribuídas a diversos fatores, como a disponibilidade hídrica, a composição química e $\mathrm{pH}$ do solo, incidência da luz solar, composição atmosférica, entre outros (28).

A avaliação da toxicidade dos extratos por A. salina, expressa em $\mathrm{DL}_{50}$, facilita a interpretação dos resultados, 
uma vez que altos valores de $\mathrm{DL}_{50}(>1000 \mathrm{ppm})$ indicam ausência de toxicidade. Além disso, esta investigação permite o direcionamento para outras atividades biológicas, como antifúngica, antimicrobiana e parasiticida (29).

Os resultados da toxicidade dos extratos foram expressos em $\mathrm{DL}_{50}$, ou seja, a concentração de extrato necessária para $\mathrm{s}$ obter cinquenta por cento de letalidade da população de larvas. $\mathrm{O}$ valor de $\mathrm{DL}_{50}$ de C. hololeuca foi $>1000$ ppm, o que aponta ausência de toxicidade neste ensaio. O valor da $\mathrm{DL}_{50}$ para Z. rhoifolium foi de $719,44 \mathrm{ppm}$, mostrando potencial efeito tóxico para este extrato. O extrato de $L$. alba apresentou valor de $\mathrm{DL}_{50}<250 \mathrm{ppm}$. Este resultado desperta atenção quanto à segurança biológica deste extrato, sinalizando presença de constituintes tóxicos.

Embora o uso popular medicinal de L. alba ocorra a partir da infusão das folhas, extratos menos polares que extratos aquosos (etanólicos e clorofórmicos, por exemplo) podem desempenhar efeitos tóxicos. $\mathrm{O}$ resultado obtido no ensaio de toxicidade preliminar com $A$. salina corrobora o resultado encontrado por Costa e cols (2004), cujo estudo mostrou que, o extrato etanólico das folhas e o extrato clorofórmico da raiz de L. alba foram os mais citotóxicos frente a linhagem de células NCI-H292 (obtida de carcinoma mucoepidermóide de pulmão humano) e HEp-2 (derivadas de carcinoma epidermóide de laringe), respectivamente (30), o que confirma a presença de compostos tóxicos nesta espécie. Neste sentido, os compostos de L. alba possivelmente responsáveis pela toxicidade devem apresentar uma polaridade média, como, por exemplo, os esteroides. Sendo assim, estudos devem ser continuados para esta espécie, a fim de realmente identificar os compostos responsáveis pela potencial toxicidade, bem como validar o uso tradicional de $L$. alba, garantindo segurança para a população.

Os resultados da atividade trombolítica in vitro dos extratos estudados encontram-se na Tabela 2. Em relação à avaliação da atividade trombolítica, as espécies $C$. hololeu$c a$ e $L$. alba mostraram baixa capacidade de lise dos coágulos, com percentual abaixo de $10 \%$. Contudo, Z. rhoifolium apresentou atividade trombolítica superior, alcançando $24,71 \%$.

As complicações decorrentes de doenças cardiovasculares foram responsáveis por cerca de quase $30 \%$ das mortes em todo o mundo no ano de 2010 (31). E uma das ferramentas utilizadas para a restauração do fluxo sanguíneo é a lise do coágulo, a partir da utilização de medicamentos trombolíticos $(10,32)$. Não são muitos os medicamentos trombolíticos utilizados clinicamente, quando comparados com outras classes de fármacos, como anticoagulantes, por exemplo (33). Sendo assim, o estudo do potencial trombolítico de extratos vegetais se faz ainda mais importante, no ensejo de encontrar novos agentes desta classe com potencial bioativo importante.

Outros autores também têm investigado o potencial trombolítico de espécies vegetais. Khatun e cols (2014) avaliaram a atividade trombolítica de Cestrum diurnum, obtendo como resultado $8,78 \%$ de lise dos coágulos (8). Por outro lado, Prasad e cols (2006) encontraram potencial promissor em duas espécies vegetais, Bacopa monnieri e Fagonia arabica, cujas atividades alcançaram os respectivos valores, $41,8 \%$ e $75,6 \%$ de lise (7).

Tabela 2: Atividade trombolítica, in vitro, de extratos extratos etanólicos de três espécies ocorrentes na Mata Atlântica: Cecropia hololeuca, Lippia alba e Zanthoxylum rhoifolium

\begin{tabular}{l|c|c|}
\hline L. alba & $9,64 \pm 1,83$ & 0,016 \\
\hline Z. rhoifolium & $24,71 \pm 10,52$ & 0,009 \\
\hline Estreptoquinase & $50,12 \pm 9,23$ & $<0,001$ \\
\hline Água destilada & $6,42 \pm 6,22$ & - \\
\hline \\
Propilenoglicol 15\% & $3,76 \pm 3,03$ & - \\
*Valores de lise expressos em média mais ou menos desvio- \\
padrão. * *Valor de $p$ ao comparar o agente trombolítico com seu \\
respectivo veículo.
\end{tabular}

Estes resultados comprovam a existência de componentes químicos em extratos vegetais com atividade trombolítica. A atividade apresentada pelo extrato de Z. rhoifolium estimula o prosseguimento da investigação no sentido de elucidar os compostos responsáveis por tal atividade. Os ensaios fitoquímicos detectaram apenas três classes químicas neste extrato: alcaloides, flavonoides e cumarinas, o que infere a possibilidade de alguma destas classes ou algum composto específico estarem diretamente ligados a esta atividade. Em linhas gerais, os compostos fenólicos são conhecidos pelo promissor potencial antioxidante. Esta propriedade biológica está diretamente associada com a prevenção e tratamento de doenças cardiovasculares, como aterosclerose e trombose $(34,35)$.

Nos extratos de L. alba e C. hololeuca foi identificada uma maior variedade de classes químicas. É possível que a variedade de compostos mascarem a atividade de algum componente específico nos respectivos extratos. Estudos de fracionamento e purificação destes extratos podem fornecer resultados mais expressivos, bem como confirmar a atividade trombolítica destas espécies.

\section{CONCLUSÃO}

Após a execução desta pesquisa, pode ser observado que as espécies estudadas possuem potencial bioativo. A toxicidade demonstrada pelo extrato de $L$. alba desperta atenção quanto à segurança no uso popular, bem como direciona a investigação de outras atividades biológicas, como antifúngica e parasiticida. O potencial trombolítico de Z. rhoifolium justifica a execução de novos ensaios químicos, a fim de elucidar os possíveis componentes responsáveis por esta atividade. Até onde é conhecido, este estudo é o primeiro relato da avaliação da atividade trombolítica destas espécies vegetais. Os resultados encontrados neste 
trabalho direcionam novas investigações para estes extratos, bem como contribuem para o conhecimento químicobiológico das respectivas espécies.

\section{AGRADECIMENTOS}

À Universidade Federal do Espírito Santo (UFES) e ao Programa de Pós-Graduação em Ciências Farmacêuticas (PPGCFAR-UFES).

\section{REFERÊNCIAS}

1. Rates SMK. Plants as source of drugs. Toxicon, 2001; 39:603-613. DOI: 10.1016/S0041-0101(00)00154-9

2. Firmo WCA, Menezes VJM, Passos CEC, Dias CN, Alves LPL, Dias ICL, Neto MS, Olea RSG. Contexto histórico, uso popular e concepção científica sobre plantas medicinais. Cadernos de Pesquisa, 2011; 18:90-95.

3. Cragg GM, Newman DJ. Biodiversidade: Um componente essencial na descoberta de fármacos. In: Yunes RA, Cechinel Filho V (Orgs.). Química de Produtos Naturais: novos fármacos e a moderna farmacognosia, 4. ed. rev. e ampl. Itajaí/Santa Catarina: Ed. da UNIVALI, 2014. p. 55-84.

4. Simões CMO, Schenkel EP. A pesquisa e a produção brasileira de medicamentos a partir de plantas medicinais: a necessária interação da indústria com a academia. Rev Bras Farmacogn, 2002; 12(1):35-40. DOI: 10.1590/S0102$695 \times 2002000100005$.

5. WHO. Herbal Medicine research and global health: an ethical analysis. Bull World Health Org, 2008; 86(8):577-656. DOI: 10.2471/BLT.07.042820

6. Di Stasi LC. Plantas medicinais: arte e ciência. Um guia de estudo multidisciplinar. São Paulo: Ed. Universidade Estadual Paulista, 1996.

7. Prasad S, Kashyap RS, Deopujari JY, Purohit HJ, Taori GM, Daginawala HF. Development of an in vitro model to study clot lysis activity of thrombolytic drugs. Thromb J. 2006; 4:14. DOI: 10.1186/1477-9560-4-14

8. Khatun A, Chowdhury UK, Jahan A, Rahman M. Cytotoxic and thrombolytic activity of the aerial part of Cestrum diurnum L. (Solanaceae). PharmacolOnline, 2014; 1:109-113.

9. Prasad S, Kashyap RS, Deopujari JY, Purohit HJ, Taori GM, Daginawala HF. Effect of Fagonia Arabica (Dhamasa) on in vitro thrombolysis. BMC Complement Altern Med, 2007; 7(36). DOI: 10.1186/1472-6882-7-36

10. Oliveira CC. Trombolíticos. Rev SOCERJ, 2001. XIV(1):47-52.

11. Collins R, Peto R, Baigent C, Sleight P. Aspirin, heparin and fibrinolytic therapy in suspected acute myocardial infarction. New Engl J Med, 1997; 336:847-860. DOI: 10.1056/NEJM199703203361207

12. Langer B, Wolosker M. Coagulação e fibrinólise: idéias atuais e suas aplicações clínicas. Rev Med (São Paulo). 2006; 85(4):157-164. DOI: 10.11606/issn.1679-9836. v85i4p157-164

13. Aleixo A, Albernaz AL, Grelle CEV, Vale MM, Rangel TF. Mudanças climáticas e a biodiversidade dos biomas brasileiros: passado, presente e futuro. Natureza e Conservação. 2010; 8(2):194-196. DOI: 10.4322/natcon.00802016
14. Campanili M, Prochnow M (Orgs.). Mata Atlântica - uma rede pela floresta. Brasília: RMA, 2006.

15. Romaniuc Neto S, Gaglioti AL. Urticaceae in Lista de Espécies da Flora do Brasil. Jardim Botânico do Rio de Janeiro. Disponível em: http://floradobrasil.jbrj.gov.br/ jabot/floradobrasil/FB15040.

16. Grandi TSM, Trindade JA, Pinto MJF, Ferreira LL, Catella AC. Plantas medicinais de Minas Gerais. Acta Bot Bras. 1989; 3(2):185-224. DOI: 10.1590/S010233061989000300018

17. Salimena FRG, Mulgura M. Lippia in Lista de Espécies da Flora do Brasil. Jardim Botânico do Rio de Janeiro. Disponível em: http://floradobrasil.jbrj.gov.br/jabot/floradobrasil/FB15171.

18. Pinto EPP, Amorozo MCM, Furlan A. Conhecimento popular sobre plantas medicinais em comunidades rurais de mata atlântica - Itacaré, BA, Brasil. Acta Bot Bras. 2006; 20(4):751-762. DOI: 10.1590/S010233062006000400001

19. Pirani JR. Zanthoxylum in Lista de Espécies da Flora do Brasil. Jardim Botânico do Rio de Janeiro. Disponível em: http://floradobrasil.jbrj.gov.br/jabot/floradobrasil/FB1162.

20. Jullian V, Bourdy G, Georges S, Maurel S, Sauvian M. Validation of use of a traditional antimalarial remedy from French Guiana, Zanthoxylum rhoifolium Lam. J Ethnopharmacol. 2006; 106:348-352. DOI: 10.1016/j. jep.2006.01.011

21. Costa AF. Farmacognosia. Lisboa: Fundação CalousteGulbenkian, $2^{\mathrm{a}}$ ed., 1982.

22. Costa AF. Farmacognosia. Lisboa: Fundação CalousteGulbenkian, $3^{\mathrm{a}}$ ed., 1986.

23. Wagner H, Bladt S. Plant drug analysis. New York: Springer Verlag, $2^{\text {a }}$ ed., 1996.

24. Meyer BN, Ferrigni NR, Putnan JE, Jacobsen LB, Nichols DE, Mclaughlin JL. Brine shrimp: a convenient bioassay for active plant constituents. Planta Med. 1982; 45:31-34.

25. Botsaris AS. Plants used traditionally to treat malaria in Brazil: the archives of Flora Medicinal. J Ethnobiol Ethnomed. 2007; 3(18). DOI: 10.1186/1746-4269-3-18

26. Aguiar JS, Costa MCCD, Nascimento SC, Sena KXFR. Atividade antimicrobiana de Lippia alba (Mill.) N. E. Brown (Verbenaceae). Rev Bras Farmacogn. 2008; 18(3):436440. DOI: 10.1590/S0102-695X2008000300018

27. Cowan MM. Plant Products as Antimicrobial Agents. Clin Microbiol. 1999; 12(4):564-582

28. Gobbo-Neto L, Lopes NP. Plantas medicinais: fatores de influencia no conteúdo de metabólitos secundários. Quím Nova. 2007; 30(2):374-381. DOI: 10.1590/S010040422007000200026 


\section{luratariona}

29. Bednarczuk VO, Verdam MCS, Miguel MD, Miguel OG. Testes in vitro e in vivo utilizados na triagem toxicológica de produtos naturais. Visão Acadêmica. 2010; 11(2):4350. DOI: $10.5380 /$ acd.v11i2.21366

30. Costa MCCD, Aguiar JS, Nascimento SC. Atividade citotóxica de extratos brutos de Lippia alba (Mill.) N.E. Brown (Verbenaceae). Acta Farm Bonaerense. 2004; 23(3):349-352.

31. Braunwald $\mathrm{E}$. What future does the pharmaceutical industry have in the cardiovascular world? Eur Heart J. 2013; 34:943-948. DOI: 10.1093/eurheartj/eht069

32. Ali MR, Hossain MS, Islam MA, Arman MSI, Raju GSR, Dasgupta P, Noshin TF. Aspect of Thromboly- tic Therapy: A review. Sci World J. 2014; 2014. DOI: $10.1155 / 2014 / 586510$

33. Cheng JWM. Updates in antiplatelet agents used in cardiovascular diseases. J Cardiovasc Pharmacol Ther. 2012; 18(6):514-524. DOI: 10.1177/1074248413499971

34. Pasknow FJ. Oxidative stress and inflammation in heart disease: do antioxidants have a role in treatment and/or prevention? Int J Inflam. 2011; 2011. DOI: $10.4061 / 2011 / 514623$

35. Freedman JE. Oxidative stress and platelets. Arterioscler Thromb Vasc Biol. 2008; 28:11-16. DOI: 10.1161/ATVBAHA.107.159178 